\title{
Small-scale structure and dynamics of the lower solar atmosphere
}

\author{
Sven Wedemeyer-Böhm ${ }^{1}$ \\ and Friedrich Wöger ${ }^{2}$ \\ ${ }^{1}$ Institute of Theoretical Astrophysics, University of Oslo, P.O. Box 1029 Blindern, N-0315 \\ Oslo, Norway; email: sven.wedemeyer@astro.uio.no \\ ${ }^{2}$ National Solar Observatory at Sacramento Peak, P.O. Box 62, Sunspot, NM 88349, USA; \\ email: fwoeger@nso.edu
}

\begin{abstract}
The chromosphere of the quiet Sun is a highly intermittent and dynamic phenomenon. Three-dimensional radiation (magneto-)hydrodynamic simulations exhibit a meshlike pattern of hot shock fronts and cool expanding post-shock regions in the sub-canopy part of the inter-network. This domain might be called "fluctosphere". The pattern is produced by propagating shock waves, which are excited at the top of the convection zone and in the photospheric overshoot layer. New high-resolution observations reveal a ubiquitous small-scale pattern of bright structures and dark regions in-between. Although it qualitatively resembles the picture seen in models, more observations - e.g. with the future ALMA - are needed for thorough comparisons with present and future models. Quantitative comparisons demand for synthetic intensity maps and spectra for the three-dimensional (magneto-)hydrodynamic simulations. The necessary radiative transfer calculations, which have to take into account deviations from local thermodynamic equilibrium, are computationally very involved so that no reliable results have been produced so far. Until this task becomes feasible, we have to rely on careful qualitative comparisons of simulations and observations. Here we discuss what effects have to be considered for such a comparison. Nevertheless we are now on the verge of assembling a comprehensive picture of the solar chromosphere in inter-network regions as dynamic interplay of shock waves and structuring and guiding magnetic fields.
\end{abstract}

Keywords. Sun: chromosphere, shock waves, MHD

\section{Introduction}

The chromosphere of the quiet Sun - a story full of misunderstandings. Apart from the ongoing controversy concerning the heating mechanism (e.g., Fossum \& Carlsson 2005), many details of the small-scale structure of the chromosphere of inter-network regions are still unknown. Already the term "chromosphere" $\dagger$ is a frequent source of misunderstandings . Certainly the large variety of phenomena observed (see, e.g., Judge 2006; Rutten 2006, 2007; De Pontieu et al. 2004) created a complex puzzle and sometimes apparent contradictions. For instance, the observed UV emission implies high temperatures, whereas the existence of carbon monoxide lines point at much cooler gas (Ayres 2002). New high-resolution observations - as reported here - show a highly dynamic and intermittent pattern that cannot be explained with the classical semi-empirical models by Vernazza et al. (1981, VAL) and Fontenla et al. (1993, FAL). Rather a time-dependent

$\dagger$ Rutten (2007, and references therein) uses the term "clapotisphere" for the shock-dominated subcanopy domain in inter-network regions, whereas his "chromosphere" refers to the fibrilar structure visible in $\mathrm{H} \alpha$ only. As "clapotisphere" stands for standing waves, we here introduce the term "fluctosphere" instead (fluctus = latin for "wave"). 

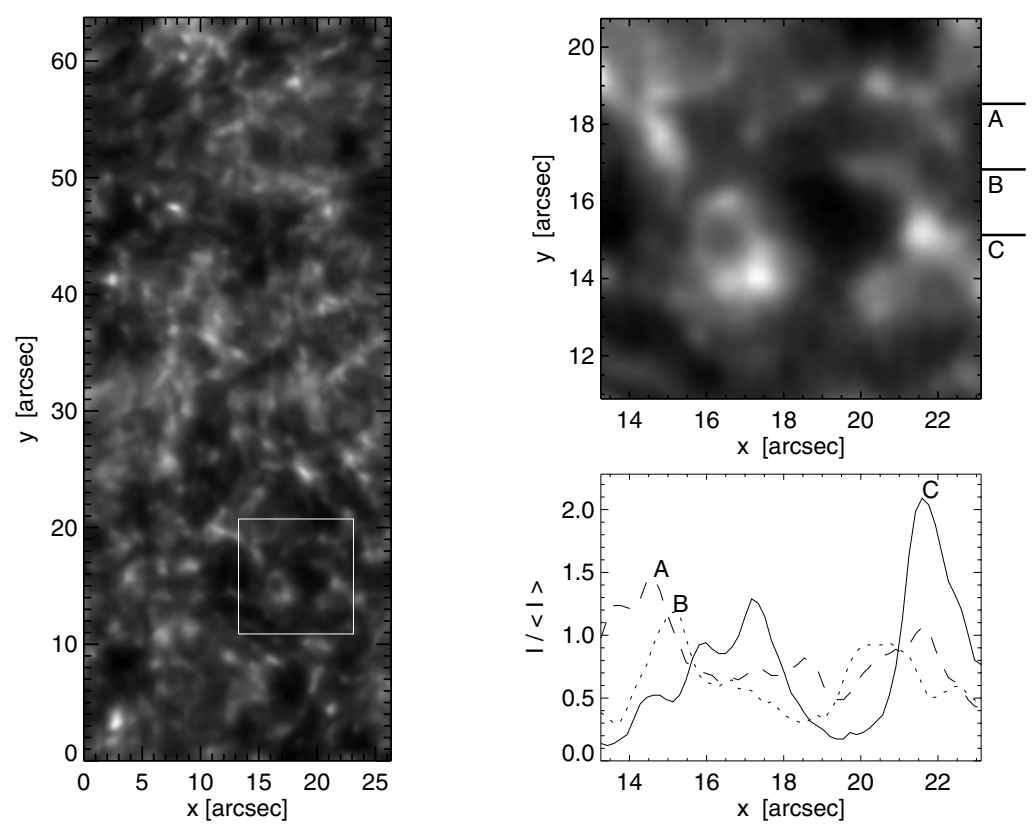

Figure 1. Single IBIS filtergram for the line core of the Ca II line at $\lambda=854.2 \mathrm{~nm}$ (left) and the close-up of the inter-network region (upper right) marked by a white square in the left image. The ticks at the right border mark the y positions of the three intensity profiles along the $\mathrm{x}$-axis, which are shown in the lower right panel.

three-dimensional model is mandatory. A self-consistent model that can fulfill all observational constraints would be most valuable for summarising the many faces of the chromosphere, indicating and understanding the most relevant processes. Chromospheric heating is a central issue as it has important implications for the atmospheres of other stellar types.

Here we report on some advances of detailed radiation magnetohydrodynamic simulations in comparison with new high-resolution observations. Some crucial aspects of such comparisons - which often result in misunderstandings - are discussed.

\section{Observations of the chromosphere}

The InterferometricBIdimensional Spectrometer (IBIS, Cauzzi et al. 2007, and references therein) at the Dunn Solar Telescope of the National Solar Observatory at Sacramento Peak is used to scan through the CaII infrared line at $\lambda=854.2 \mathrm{~nm}$ in (2D) spectropolarimetric mode. Images at 17 wavelength positions in the line wing and in the line core are taken, resulting in a overall cadence of $28 \mathrm{~s}$. The field of view is 26.5 " $\times 64$ "; the pixelscale is 0.17 " / px. Channels for continuum, G-band, and $\mathrm{H} \alpha$ are used simultaneously in addition to IBIS.

The Ca II line core image for at $\lambda=854.2 \mathrm{~nm}$ in Fig. 1 features a bright mesh-like pattern with dark regions inbetween. The spatial scales of the pattern are similar to the granulation. Next to the known magnetic network cells also a small-scale pattern is visible in the inter-network regions (see upper right panel). It exhibits ring-like structures and very short-lived bright points. The profiles (lower right panel) show a large intensity variation and very small minimum values. Image sequences reveal that the small-scale 


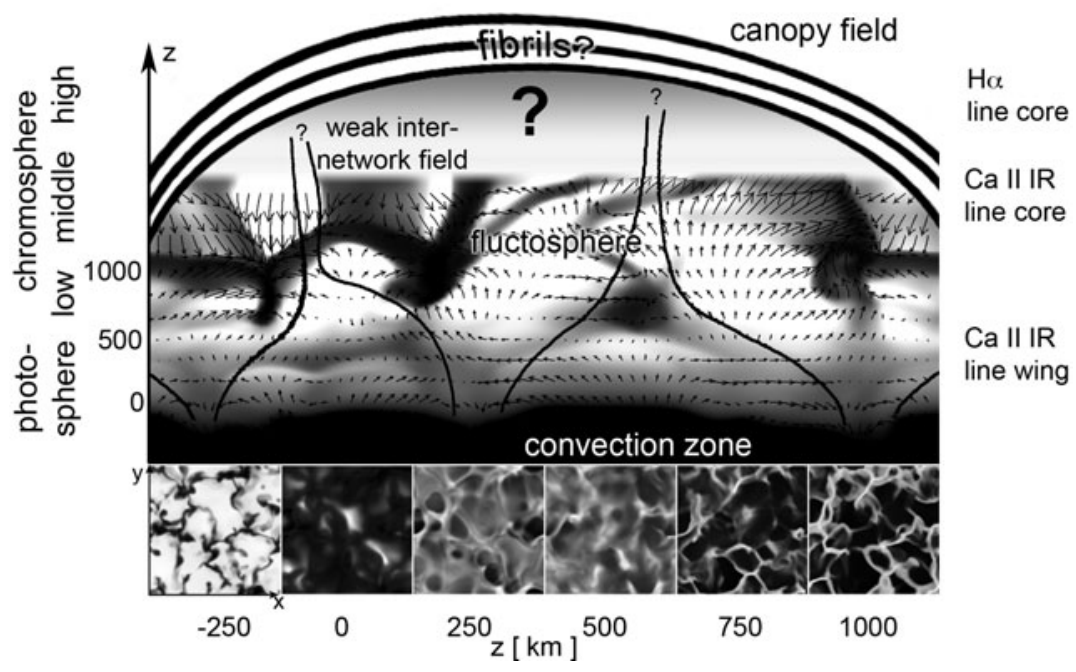

Figure 2. Upper panel: Schematic structure of the lower atmosphere in quiet inter-network regions. Velocity field (arrows) and gas temperature (color-coded: white $=$ cool, black=hot) based on the model by Wedemeyer et al. (2004, W04). The lines represent magnetic field lines, forming a canopy (thick) and a weak-field "small-scale canopy" (thin) in the inter-network region below. On the right the rough (anticipated) formation height ranges of some diagnostics are indicated. Lower panels: Horizontal cross-sections at different heights from the model by W04. Integral components of the low inter-network atmosphere are the granulation at the bottom of the photosphere $(z \sim 0 \mathrm{~km})$, the reversed granulation produced by convective overshooting $(z \sim 250 \mathrm{~km})$, a layer with little fluctuations near the height of the classical temperature minimum $(z=500 \mathrm{~km})$, and the fluctosphere $(z>700 \mathrm{~km})$ produced by upward propagating and interacting shock waves. The structure of the blank layer marked with "?", i.e. the interface between fluctosphere and canopy domain, is still poorly known as it demands for a sophisticated non-LTE modelling. Please note that the temperature in the lower panels is scaled individually. The variation at $z=500 \mathrm{~km}$ is much smaller than in the other layers.

pattern is evolving much faster than the granulation and reversed granulation in the photosphere below. In particular the bright points only "flash up" for a short moment. These new observations support the results reported by Wöger et al. (2006). See also Cauzzi et al. (2007), Tritschler et al. (2007), and Reardon et al. (2007). We again interpret the observation as manifestation of the interaction of propagating shock waves. The bright points are in this picture the collision points of neighbouring wave fronts.

\section{Numerical simulations}

The 2D/3D numerical simulations considered here all comprise a small part of the surface-near layers and vertically extent from the upper convection zone to the middle chromosphere. The hydrodynamical model(s) by Wedemeyer et al. (2004), computed with $\mathrm{CO}^{5}$ BOLD (Freytag et al. 2002), feature(s) a small-scale chromospheric pattern, which consists of hot shock fronts and intermediate cool post-shock regions (see Fig. 2). It is caused by the propagation and interaction of shock waves that are excited in the layers below. Due to adiabatic expansion of the post-shock regions the gas temperature reaches values down to $2000 \mathrm{~K}$ in the model chromosphere. An obvious example of a post-shock region can be seen in the left part of Fig. 2. The corresponding strong shock front "collides" with the neighbouring front to the left, compressing the gas in the region in-between and rising its temperature. The typical spatial scale is similar to the granular one. The pattern changes dynamically on typical time scale of $\sim 30 \mathrm{~s}$. The 
magnetohydrodynamic model by Schaffenberger et al. (2006) and also the models by Skartlien et al. (2000) and Hansteen \& Gudiksen (2005) are very similar concerning structure and dynamics. Also the magnetic field in the model chromospheres is highly dynamic. A look at horizontal cross-sections at different heights (see, e.g., Fig. 1 in Schaffenberger et al. 2006) implies that the chromospheric field in inter-network regions is much weaker $(|B|<50 \mathrm{G})$ than the photospheric one but evolves much faster. The compression and expansion induced by the ubiquitous propagating shocks also play an important role for the small-scale structure of the weak inter-network field (see Fig. 4 in Schaffenberger et al. 2005). Figure 2 shows a possible combination of the resulting weak field and an overlying stronger magnetic canopy, which is rooted in the network boundaries. A similar picture can be seen from other new simulations, e.g., by Leenaarts et al. (2007, see Fig. 1 therein).

The simulations and also the observations presented in the previous sections both indicate that the chromospheric inter-network regions of the quiet Sun are highly dynamic with inhomogeneities on small temporal and spatial scales. This behaviour, which was already suggested by Carlsson \& Stein (1995), certainly has important consequences for the modelling of physical processes. An important example is the ionisation of hydrogen. Recent 2D and 3D non-equilibrium simulations by Leenaarts \& Wedemeyer-Böhm (2006) and Leenaarts et al. (2007) confirm the earlier conclusions by Carlsson \& Stein (2002) that the ionisation degree and the electron density are fairly constant over time and space and tend to be at values set by hot propagating shock waves. In contrast, the ionisation degree varies by more than 20 orders of magnitude between hot, shocked regions and cool, non-shocked regions when assuming instantaneous equilibrium. Obviously such deviations from equilibrium must be taken into account for the physical description of the dynamic solar chromosphere. Another important consequence is that the cool intermediate phases leave almost no trace in the ionisation degree, which certainly complicates the observational proof of their existence.

\section{Comparison of simulations and observations}

On the way towards a comprehensive model of the solar atmosphere, detailed comparisons between observations and numerical simulations are needed to check if and what physical ingredients are missing and what aspects are already modelled realistically. In the case of the chromosphere, particular attention has to be paid to the energy balance and the gas temperature amplitudes. Kalkofen (2003b, 2005) claims that the temperature amplitudes in the dynamical models are too large compared to "modeling based on the emergent spectrum", where the latter refers to semi-empirical models by VAL and FAL. Their models only show "modest fluctuations $(\delta T \sim 300 \mathrm{~K})$ " (Kalkofen 2003b). Kalkofen (2003a) argues that the assumption of static atmospheres by VAL and FAL is "justified by the small temperature differences between the models which remain below about $5 \%$ of the average temperature." This argument is obviously misleading as it is only the difference between averages (of different atmospheric regions/brightness components represented by individual VAL models), which consequently refers to variations on very large spatial scales and should not be interpreted as the temperature fluctuation introduced by the propagation of waves on small spatial scales (say $\Delta x<2000 \mathrm{~km}$ ). The existence of strong temperature gradients on small spatial scales does not contradict the models of VAL and FAL if these are interpreted as average stratifications only. Rather the dynamical models can produce a VAL-like average chromospheric temperature rise when giving a higher weight to the large temperatures in shock waves (Carlsson \& Stein 1995; Wedemeyer et al. 2004). 


\subsection{Gas temperature and emergent intensity - A few words of caution}

One must not quantitatively compare observed intensities with the gas temperature in a model chromosphere. In particular, just estimating gas temperatures by eye from horizontal cross-sections at different heights (say with a $\Delta z=250 \mathrm{~km}$ ) is certainly an inadequate approach. Gas temperature cannot directly be interpreted as intensity and vice versa because (i) the intensity does not originate from a fixed infinitesimal thin layer but from an extended height range and (ii) (at least in case of the chromosphere) "the convenient approximation of LTE no longer holds for the calculation of radiative energy transfer" (Kalkofen 2004).

A look in any textbook on radiative transfer clearly shows that opacity and source function do not only depend on the gas temperature but also on parameters as, e.g., gas and electron density, chemical composition and ionisation stage of the gas. In particular the electron density and ionisation degree are subject to deviations from equilibrium in most of the atmosphere (see Sect. 3) and thus cannot be described in LTE. The chromospheric gas density is modulated by compression and expansion due to propagating waves, while, e.g., the population densities of atomic energy levels depend directly on the (non-local!) radiation field itself. Even in a simple case the emergent intensity is always the result of the integration of contributions along the line of sight. It consequently represents the integrated (thermodynamic) conditions of an extended formation height range and not the local conditions at a particular height. In an inhomogeneous intermittent chromosphere the true thermal structure is thus obscured by an intrinsic "smearing" along the optical depth $\tau$.

A comparison of observations and models is thus complicated by the fact that the gas temperature can be investigated at individual geometrical heights in the models, whereas observed intensity always originates from an extended formation height range. In some cases the corresponding contribution functions even have multiple peaks at different heights, making the use of the term "formation height" problematic. This is an intrinsic physical problem that cannot be removed. One can only try to minimize the effect by choosing a suitable diagnostic (with small and well-defined variation in formation height) in combination with the detailed comparison by means of non-LTE radiative transfer calculations based on forward modelled simulations.

In addition the observations are technically limited by the attainable resolution in angle $\Delta \alpha$, time $\Delta t$, and wavelength $\Delta \lambda$ :

(a) Atmospheric seeing and instrumental effects limit the spatial scales accessible in observations and smear out features on small scales.

(b) The formation height ranges vary significantly in time and space so that intensities refer rather to corrugated surfaces of optical depth instead of plane horizontal cuts.

(c) A limited spectral resolution, e.g. when using a broad band filter, essentially mixes together intensity contributions from different height ranges, effectively smearing out the small-scale structure of the atmosphere. For very narrow filters Doppler-shifts have to be considered.

In contrast to the unavoidable intrinsic smearing in $\tau$, advances in observational technique have the potential to reduce the smearing in $\alpha, t$, and $\lambda$. The combination of these effects, however, can prevent the detection of a cold and diluted post-shock region behind a shock wave with current and past instruments, as such regions are quite small and exist for a short time only. Consequently empirically derived temperature amplitudes bear the danger of being systematically too low. An example might be the low values suggested by Avrett et al. (2006) and Avrett (2007), which rely on SUMER observations (Wilhelm et al. 2005) with limited spatial resolution. 


\subsection{CaII IR line}

For a detailed comparison with the CaII data presented here (see Sect. 2) synthetic intensity images still need to be calculated. This task, although very involved, is currently in progress. On the observational side, Doppler-shifts move too fast propagating features outside of the filter range. This effect can be corrected for as the IBIS scan includes the neighbouring wavelength positions at only small delay. (A detailed publication is currently in preparation.)

Nevertheless the general picture exhibited by observations and simulations is astonishingly similar. Both show the chromosphere as highly dynamic and intermittent phenomenon. A quantitative comparison by means of non-LTE radiative transfer calculations, however, will always be hampered by the extended formation height range and non-equilibrium effects. The conclusions might thus be of limited value for our understanding of the quiet Sun chromosphere.

\section{3. (Sub-)millimetre continua}

A very promising alternative are observations in the (sub-)millimetre range with the Atacama Large Millimeter Array (ALMA) - an array of 50 antennae with diameters of $12 \mathrm{~m}$ on a plateau at $5000 \mathrm{~m}$ altitude in the Chilean Andes. It will commence full operation in 2012. Wavelengths in the range of 0.3 to $3.6 \mathrm{~mm}$ will be accessible. Synthetic brightness temperature maps have been calculated by Wedemeyer-Böhm et al. (2007). The maps exhibit very similar spatial and temporal scales than the original gas temperature in the model chromosphere, which is closely mapped with this kind of diagnostic. When using the non-equilibrium electron densities from the simulation by Leenaarts \& WedemeyerBöhm (2006, see Sect. 3) as an input for intensity synthesis, the formation height range stays on average very similar but varies less in the non-equilibrium approach. The resulting brightness temperature is even closer to the gas temperature at a fixed geometrical height plane, which simplifies the interpretation. In either case the formation height increases with wavelength $\lambda$ and heliocentric position $(\mu=\cos \theta)$ from centre to limb. Consequently the sampled layer can more or less be chosen by $\lambda$ and $\mu$, facilitating a tomography of the three-dimensional atmospheric structure.

\section{Assembling a new picture of quiet Sun inter-network regions}

Another source of confusion might arise from assigning fixed formation height ranges to the different diagnostics (see, e.g., VAL). Considering a substantial overlap of formation height ranges of the individual diagnostics and a significant variation in space and time (caused by "fluctospheric" shock waves, see Carlsson \& Stein 1998), the different observational constraints can be assembled to a comprehensive picture of the quiet Sun atmosphere as, e.g., suggested by Rutten (2007, see also Fig. 2 in this article). It might be so that the emission features like the one near $117 \mathrm{~nm}$ (S I, Avrett et al. 2006) but also the He II line at $164 \mathrm{~nm}$ (Wahlstrom \& Carlsson 1994) are formed above/in the magnetic canopy layer, while the strong shock activity, which, e.g., produces bright points observed in the Ca II lines, is limited to the "fluctosphere" regions below the canopy (see Fig. 2). The canopy field certainly modifies the properties of upward propagating disturbances (cf., e.g., Carlsson et al. 1997; Bogdan et al. 2003), causing distinct differences in structure and dynamics of the sub-canopy and the canopy domain. It is reasonable to assume that the $\mathrm{H} \alpha$ line core tends to be formed higher up in the atmosphere than the cores of the Ca II lines (cf. Langangen et al. 2007). Indeed a closer look at the high-resolution H $\alpha$ line core observations by Rouppe van der Voort et al. (2007) not only reveals a wealth of fibrils covering the inter-network regions but also the wave-generated "fluctosphere" 
shining through at locations where the fibrils are (partially) transparent. To some extent the scene reminds of floating spaghetti pushed around by boiling water underneath. Depending on the structure and strength of the magnetic (canopy) field, fibrils can show up in the line cores of the CaIR lines - provided that a very narrow filter is used (see, e.g., Fig. 5 by Cauzzi et al. 2007).

\section{Conclusions and Outlook}

State-of-the-art numerical simulations exhibit a highly dynamic chromosphere, which is characterised by propagating and interacting shock waves with co-existing hot and cool regions. New observations, as presented here, now have a sufficiently high spatial, temporal and spectral resolution to resolve a pattern of bright structures and dark regions. As synthetic intensity images for the cores of the prominent Ca II lines are still missing, these observations can only be compared to the simulations on a qualitative basis at the moment. Nevertheless they clearly support the picture of the chromosphere as highly dynamic and intermittent phenomenon like it was already implied by the pioneering simulations by Carlsson \& Stein (1995, 1998). Even Kalkofen (2004) stated that "[d]etailed observations show the chromosphere to be highly dynamic". The model atmospheres by VAL and FAL, although certainly very elaborate, suffer from the basic assumption of a one-dimensional stratified static atmosphere. This assumption is clearly questioned by recent high-resolution observations. Strong intensity fluctuations are now observed although one still should need be careful with deriving statements concerning the gas temperature. Frequently it is argued that the amplitudes and minimum values of gas temperature in the model atmospheres are not observed and that they are wrong as they do not agree with the VAL models (cf. Kalkofen 2003b). These arguments obviously do not hold any longer in view of new observational results. VAL-type atmospheres should thus be considered as qualitative averages, which could at best be interpreted as variations on large spatial scales. Instead of aiming at an agreement with VAL models, modern 3D radiation (magneto-)hydrodynamical simulations must be directly compared to observations. Certainly the quantitative values of the temperature fluctuations in these models (apart from the low to middle photosphere) still suffer from the simplified treatment of radiative transfer and resulting uncertainties in the energy balance, which are, however, a necessary compromise in order to keep the computations tractable.

The next steps towards realistic chromosphere models requires more work on the modelling of non-equilibrium effects, which are important for the energy balance and thus the temperature amplitudes in the chromosphere. An efficient non-LTE radiative transfer scheme is a major goal.

Observational emphasis should be given to (i) continued aiming at a combination of high spatial and temporal and spectral resolution, as they are crucial for a meaningful comparison and interpretation, and (ii) the development of new diagnostics as, e.g., the (sub-)millimetre continua. Especially the upcoming ALMA might allow a tomography of the solar atmosphere, finally revealing details of its three-dimensional structure.

\section{Acknowledgements}

We would like to thank the organisers of the International Astronomical Union Symposium 247 for an interesting meeting. SWB is grateful to Vilfredo for stimulating input. He was supported by the Research Council of Norway, grant 170935/V30. We thank M. Carlsson, R. Rutten, L. Rouppe van der Voort, Ø. Langangen, A. Tritschler, and $\mathrm{K}$. Reardon for discussions. NSO is operated by the Association of Universities for Research in Astronomy, Inc (AURA), for the National Science Foundation. 


\section{References}

Avrett, E. H. 2007, in Astronomical Society of the Pacific Conf. Series, Vol. 368, The Physics of Chromospheric Plasmas, ed. P. Heinzel, I. Dorotovič, \& R. J. Rutten, 81

Avrett, E. H., Kurucz, R. L., \& Loeser, R. 2006, A\& A, 452, 651

Ayres, T. R. 2002, ApJ, 575, 1104

Bogdan, T. J., Carlsson, M., Hansteen, V. H., et al. 2003, ApJ, 599, 626

Carlsson, M., Judge, P. G., \& Wilhelm, K. 1997, ApJL, 486, L63

Carlsson, M. \& Stein, R. F. 1995, ApJL, 440, L29

Carlsson, M. \& Stein, R. F. 1998, in IAU Symposium, Vol. 185, New Eyes to See Inside the Sun and Stars, ed. F.-L. Deubner, J. Christensen-Dalsgaard, \& D. Kurtz, 435

Carlsson, M. \& Stein, R. F. 2002, ApJ, 572, 626

Cauzzi, G., Reardon, K. P., Uitenbroek, H., et al. 2007, ArXiv e-prints, 709

De Pontieu, B., Erdélyi, R., \& James, S. P. 2004, Nature, 430, 536

Fontenla, J. M., Avrett, E. H., \& Loeser, R. 1993, ApJ, 406, 319 (FAL)

Fossum, A. \& Carlsson, M. 2005, Nature, 435, 919

Freytag, B., Steffen, M., \& Dorch, B. 2002, Astron. Nachr., 323, 213

Hansteen, V. H. \& Gudiksen, B. 2005, in ESA Special Publication, Vol. 592, Solar Wind 11/SOHO 16, Connecting Sun and Heliosphere

Judge, P. 2006, in Astronomical Society of the Pacific Conf. Series, Vol. 354, Solar MHD Theory and Observations: A High Spatial Resolution Perspective, ed. J. Leibacher, R. F. Stein, \& H. Uitenbroek, 259

Kalkofen, W. 2003a, in ASP Conf. Series, Vol. 286, Current Theoretical Models and Future High Resolution Solar Observations: Preparing for ATST, ed. A. Pevtsov \& H. Uitenbroek, 443

Kalkofen, W. 2003b, in Bulletin of the American Astronomical Society, Vol. 35, 826

Kalkofen, W. 2004, in IAU Symposium, Vol. 219, Stars as Suns : Activity, Evolution and Planets, ed. A. K. Dupree \& A. O. Benz, 115

Kalkofen, W. 2005, in ESA SP, Vol. 560, 13th Cambridge Workshop on Cool Stars, Stellar Systems and the Sun, ed. F. Favata \& et al., 695

Langangen, O., Carlsson, M., Rouppe van der Voort, L., Hansteen, V., \& De Pontieu, B. 2007, ArXiv e-prints, 710

Leenaarts, J., Carlsson, M., Hansteen, V., \& Rutten, R. J. 2007, AछA, 473, 625

Leenaarts, J. \& Wedemeyer-Böhm, S. 2006, A\&A, 460, 301

Reardon, K. P., Cauzzi, G., \& Rimmele, T. 2007, in Astronomical Society of the Pacific Conf. Series, Vol. 368, The Physics of Chromospheric Plasmas, ed. P. Heinzel, I. Dorotovič, \& R. J. Rutten, 151

Rouppe van der Voort, L. H. M., De Pontieu, B., Hansteen, V. H., Carlsson, M., \& van Noort, M. 2007, ApJL, 660, L169

Rutten, R. J. 2006, in ASP Conf. Series, Vol. 354, Solar MHD Theory and Observations: A High Spatial Resolution Perspective, ed. J. Leibacher, R. F. Stein, \& H. Uitenbroek, 276

Rutten, R. J. 2007, in Astronomical Society of the Pacific Conf. Series, Vol. 368, The Physics of Chromospheric Plasmas, ed. P. Heinzel, I. Dorotovič, \& R. J. Rutten, 27

Schaffenberger, W., Wedemeyer-Böhm, S., Steiner, O., \& Freytag, B. 2005, in ESA SP, Vol. 596, Chromospheric and Coronal Magnetic Fields, ed. D. E. Innes, A. Lagg, \& S. A. Solanki

Schaffenberger, W., Wedemeyer-Böhm, S., Steiner, O., \& Freytag, B. 2006, in Astronomical Society of the Pacific Conf. Series, Vol. 354, Solar MHD Theory and Observations: A High Spatial Resolution Perspective, ed. J. Leibacher, R. F. Stein, \& H. Uitenbroek, 345

Skartlien, R., Stein, R. F., \& Nordlund, Å. 2000, ApJ, 541, 468

Tritschler, A., Schmidt, W., Uitenbroek, H., \& Wedemeyer-Böhm, S. 2007, A\&A, 462, 303

Vernazza, J. E., Avrett, E. H., \& Loeser, R. 1981, ApJS, 45, 635 (VAL)

Wahlstrom, C. \& Carlsson, M. 1994, ApJ, 433, 417

Wedemeyer, S., Freytag, B., Steffen, M., Ludwig, H.-G., \& Holweger, H. 2004, A\&\&A, 414, 1121

Wedemeyer-Böhm, S., Ludwig, H. G., Steffen, M., Leenaarts, J., \& Freytag, B. 2007, A $\& A$, 471, 977

Wilhelm, K., Schühle, U., Curdt, W., et al. 2005, A\&A, 439, 701

Wöger, F., Wedemeyer-Böhm, S., Schmidt, W., \& von der Lühe, O. 2006, A\&\&A, 459, L9 